\title{
The Radio-Opaque Appendicolith — Its Significance in Clinical Practice
}

\author{
Maj P J Guy
}

FRCS, RAMC

Capt C A Pailthorpe

$M B, C h B, R A M C$

British Military Hospital, Hong Kong

SUMMARY: Four cases are reported, in whom radioiogically opaque appendiceal coproliths (appendicoliths $\vec{P}$

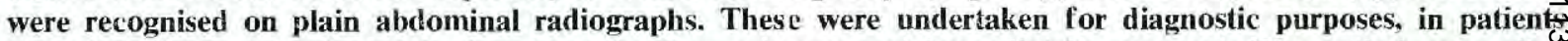
with right-sided abdominal pain, in whom the diagnosis was not immediately apparent. The pathology, radiog logical features, and clinical significance of the phenomenon are discussed, and the association of appendi⿱乛龰 coliths with appendiceal perforation and gangrene is stressed. The fact that clinical signs and symptomo of acute appendicitis are often misleading and sometimes minimal, even in the presence of severe local peritoneal infection is of importance to physicians as well as surgeons.

\section{Case Reports}

Case 1: A Caucasian boy aged eight was admitted with a one week history of severe watery diarrhoea and vomiting, with intermittent central and right-sided abdominal pain. immediately prior to admission he developed severe right loin and right-sided abdominal pain, and a pyrexia of $40^{\circ} \mathrm{C}$. On examination, he was cxtremely toxic, and was markedly tender in the right renal area. Although tender elsewhere over the right side of the abdomen, there was little guarding and no rebound tenderness. There were no pus cells in the urine and a white cell count revealed a neutrophil leucocytosis of 20.2. A plain abdominal radiograph was undertaken and a classical appendicolith was discovered (Fig. 1). Due to the marked localisation of symptoms and signs, a diagnosis of perinephric abscess was made, presumed to be secondary to a high retrocaccal appendicitis. Through a loin incision, a large retroperitoneal collection of pus was drained and the patient made an uneventful recovery. Six weeks later, a retrocaecal appendix was removed at interval appendicectomy and the appendicolith was radiologically confirmed within the specimen.

Case 2: A 19 year old Chinese male, was admitted under the care of the physicians, with a 15 day history of right hypochondrial pain, nausea and diarrhoea, On admission, he was apyrexia and appeared faintly jaundiced. On abdominal examination, there was right subcostal tenderness, but no hepatomegaly. Additionally, some tenderness in the right loin and right iliac fossa was noted, but there was no guarding or rebound tenderness. Liver func-

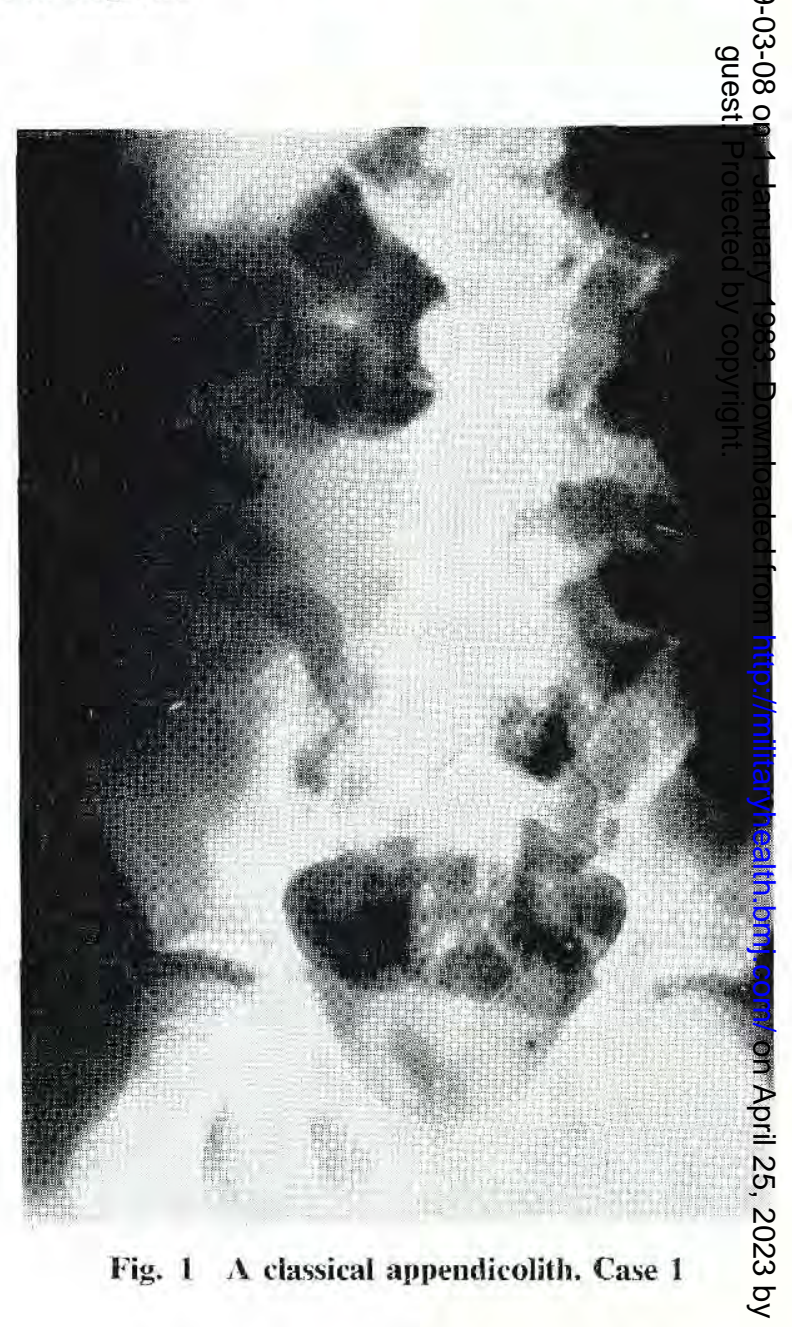


tion tests (bilirubin 72, alkaline phosphatase 265 , SGOT 119, SGPT 373) together with the abdominal findings, were felt to be compatible with a provisional diagnosis of infective hepatitis. After 24 hours. he developed a high fever and increasing pain in the right loin and right iliac fosa, together with guarding and rebound tenderness over the right side of the abdomen. A plain abdominal radiograph revealed an appendicolith (Fig. 2). At subsequent appendicectomy, a retrocaccal gangrenous, perforated appendix was found and the patient made an uneventful recovery.

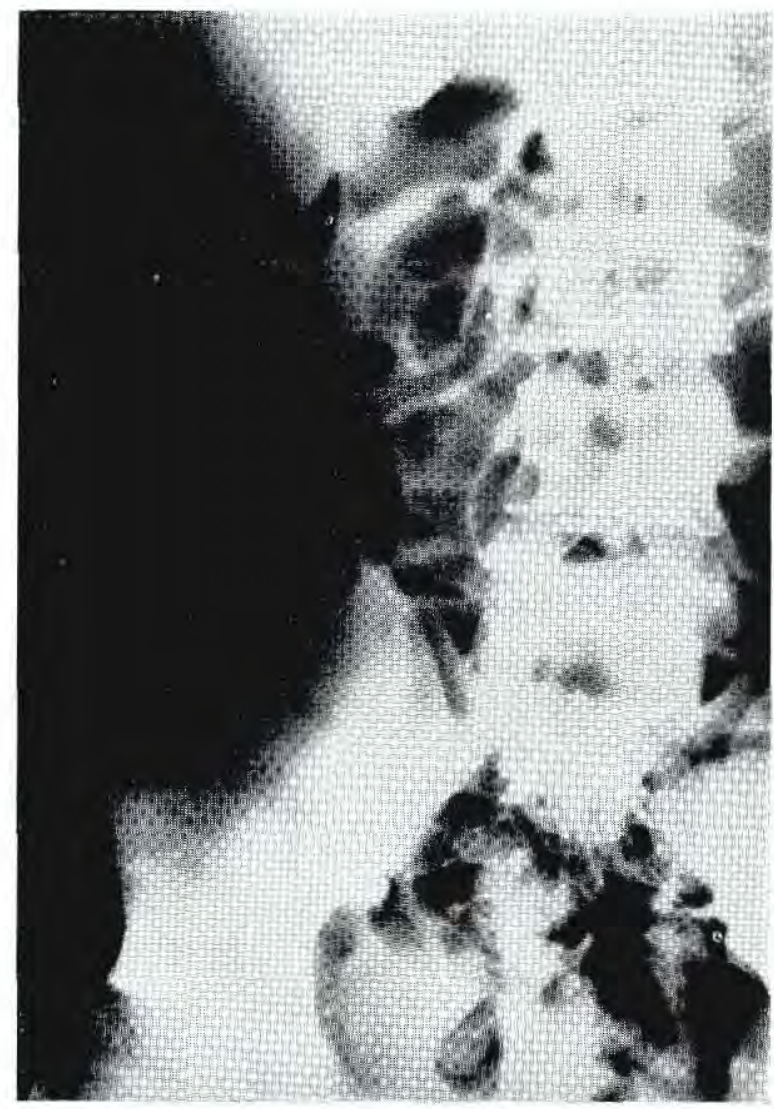

Fig. 2 An appendicolith. Case 2

Case 3: A 14 year old Nepali boy, was admitted with a three day history of vague central abdominal pain, localising in the right iliac fossa. On admission, the abdominal signs were those of early acute appendicitis. A plain abdominal radiograph revealed an appendicolith (Fig. 3). At appendicectomy, an un-

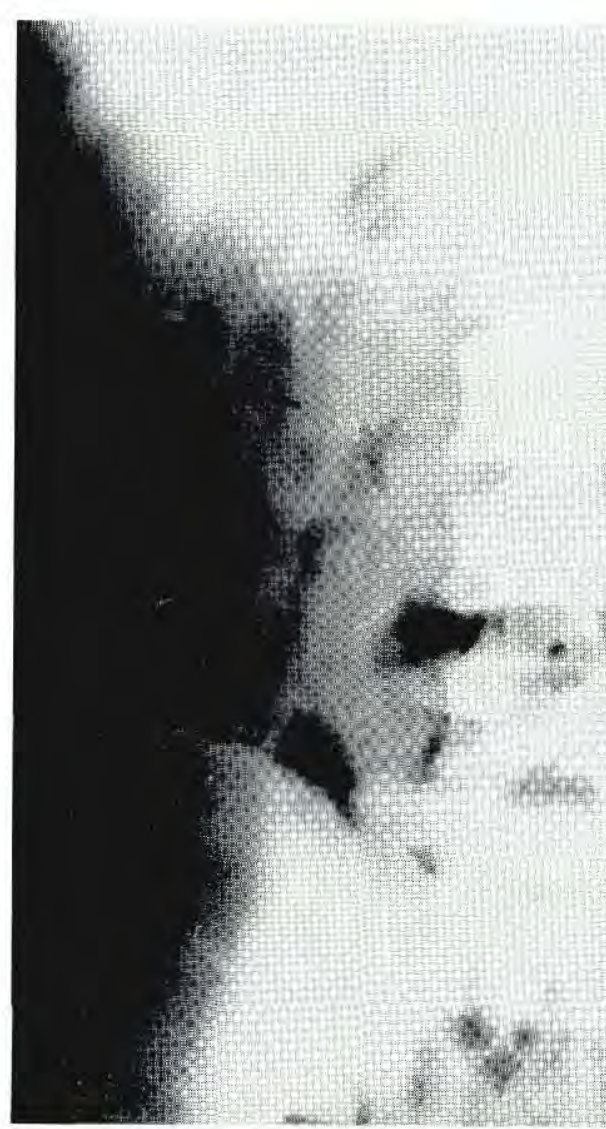

Fig. 3 An appendicolith. Case 3

expeciedly perforated and gangrenous preileal appen $\overrightarrow{\overline{\hat{O}}}$ dix was associated with marked local suppurative peritonitis.

Case 4: A 43 year old Chinese male, was admitted under the care of the physicians with an 18 day history of general malaise, loose stools and vaguef right hypochondrial and right iliac fossa pain. Orw examination, he had a fever of $39^{\circ} \mathrm{C}$ and was markedly tender in the right hypochondrium ands over the right renal area. There was no guarding of rebound tenderness in the right iliac fossa. White cell count revealed a leucocytosis of 20.0. Liver function tests were normal. Clinically, he was felo to have acute cholecystitis, and was treated con- $\rightarrow$ servatively for 24 hours with oral fluids and anti을. biotics. A repeat examination revealed a vague N tender mass high in the right iliac fossa, and a sub=r sequent plain abdominal radiograph revealed a larg calcified opacity, identified as an appendicolith, 


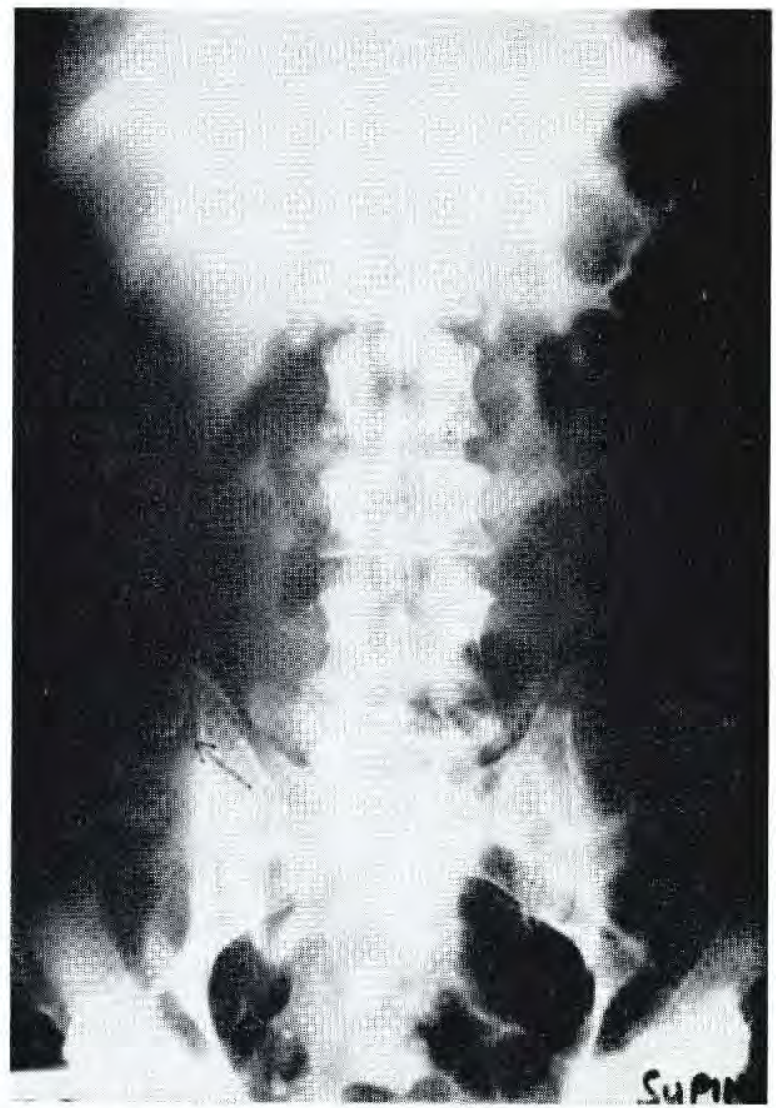

Fig. 4 Large calcified opacity. Case 4

(Fig. 4). The patient was diagnosed as having an appendix mass, and settled uneventfully on intravenous fluid replacement, ampicillin and metronidazole. At interval appendicectomy three months later, a long retrocaecal appendix containing an appendicolith was removed from a mass of fibrous tissue.

\section{Discussion}

Our small series of four appendiceal coproliths demonstrates three important characteristics of the clinical presentation of appendicolith associated appendicitis.

(1) There is a marked association between appendicoliths and severe, complicated appendicitis.

(2) In three of our cases, severe appendiceal inflammation in a retrocolic position produced confusing symptoms and signs. This led to a delay in the correct diagnosis of about 24 hours until plain abdominal radiographs were undertaken and the appendiceal coprolith recognised.
(3) The symptoms and signs in the remaining case were surprisingly slight, bearing in $\min \nsubseteq$ the severity of inflammation encountered an operation.

\section{Pathology}

Kelly and Hurdon ${ }^{1}$ and Wangensteen and Bowers? provided the early descriptions of the pathologicaf processes involved. The appendix behaves as ate atonic closed loop. Faecal particles within the lumer fail to return to the main stream, become inspissatec and form a focus of irritation to the appendiceap mucosa. This responds by increasing mucous outpul $\overrightarrow{0}$ the mineral salt content of which becomes deposited. on the faecal 'island.' These salts, primarily calciunf్ and phosphorous, increase the size of the mass thus exacerbating the irritation and thereby pers petuating the cycle. Eventually the lumen becomeş blocked by the enlarging appendicolith, and clinican appendicitis supervenes.

\section{Radiology}

In Berg and Berg's series ${ }^{3}$ approximately $90 \%$ all coproliths were demonstrated in patients under the age of 40 years, a factor having obvious sighi ficance to Army clinicians. Typically, they are rot巴 or oval. Frequently they are laminated, but may $\frac{Q}{9}$ homogenous or mottled, when they can be diffiglt to spot amidst faecal shadows. Rarely, they ar multiple. Shaham reporting one case of 23 appo dicoliths in a single appendix ${ }^{4}$. They are usuaty found in the right lower quadrant but frequently the right upper quadrant. Occasionally they are seero elsewhere, reflecting the varying anatomical posio tions seen at appendicectomy. They may be con $\mathbb{Q}$ fused with gall stones, ureteric or vesical calculi $\underset{F}{\vec{F}}$ phleboliths or calcified mesenteric lymph nodes, bu these are rarities in very young patients. Other causes of confusion are bone islands or radio-opaque्ठ tablets.

\section{Clinical features}

Correctly identifying the presence of an appen $-\frac{3}{2}$ diceal opacity may clinch the diagnosis of acute巾 appendicitis at an early stage. In young children, in whom the diagnosis of appendicitis can be particu-o larly difficult, it may be of great value. In theif. series, Wilkinson et $\mathrm{al}^{5}$ demonstrated appendicoliths in eight out of $41(32 \%)$ infants under the age of two years with acute appendicitis. More significantly in this series, six out of these eight appendices were perforated at the time of surgery and this is in agree? ment with others who have demonstrated a high rato of perforation in appendicolith associated appendi= citis e.g. 32 out of 34 in Brady and Carroll's series " $N$ Williams $^{7}$ also noted that about $50 \%$ of young 
patients would not have had sufficient clinical evidence to merit exploration without the presence of an appendicolith, and this figure was identical in Berg and Berg's series ${ }^{3}$. Thus, appendicolith related perforations would appear to be a relatively silent and therefore dangerous phenomenon in some instances, a strong indication for careful scrutiny of abdominal radiographs, event in the presence of mild abdominal symptoms and signs. Indeed, Copeland and Long ${ }^{8}$ go so far as to state their belief that a patient with abdominal pain and a detectable calcified appendicolith has a $90 \%$ chance of having appendicitis, and on this basis they make a strong plea for elective appendicectomy for appendiceal calculus in an asymptomatic patient.

Our own small series should act as a reminder of this important radiological feature in resolving the differential diagnosis of abdominal pain. Appendicitis is sometimes a fatal disease, approximately four per million of the population of England and Wales dying of th: disease in 1981. Twelve people under 35 years of age died of generalised peritonitis following perforation of the appendix, and any investigation which might reduce this toll must be well worth while. (OPCS mortality statistics 1981.)

\section{REFERENCES}

1 Killy $H$ A AND Hurdon E. The Vermiform Appendix. Philadelphia. W B Saunders \& CO. 1905.

2 Wangensteen $O H$ and Bowers W $F$. The sig竎ficance of the Obstructive Factor in the Genesis of Acute Appendicitis. Arch Surg 1937; 34: 496-5.

3 BERG R M AND BERG H M. Coproliths. Radiolo $\frac{5}{8} y$ 1957; 68: 839-844.

4 Shahar J. An Unusual Case of Multiple Appe하diceal Lithiasis. Radiology 1940; 35: 89-90.

5 Wilkinson R H, Bartlett R $\mathbf{H}$ and ERAKLis A 9 . Diagnosis of Appendicitis in Infancy - The Valcoe of Abdominal Radiographs. Amer I Dis Child 1950; 118: 687-59J.

6 Brady B M ANd Carroll D S. The Significan $\overrightarrow{\mathrm{C}}$ of the Calcified Appendiceal Enterolith. Radiology 1957; 68: 648-653.

7 Williams H J. Coproliths in Children. Paediatr胥s 1964; 34.

8 Copeland E M ANd Long J M. Elective Append cectomy for Appendiceal Calculus. Surg Gynec and

9 Obstet 1970; 130: 439-442. Table 1 .

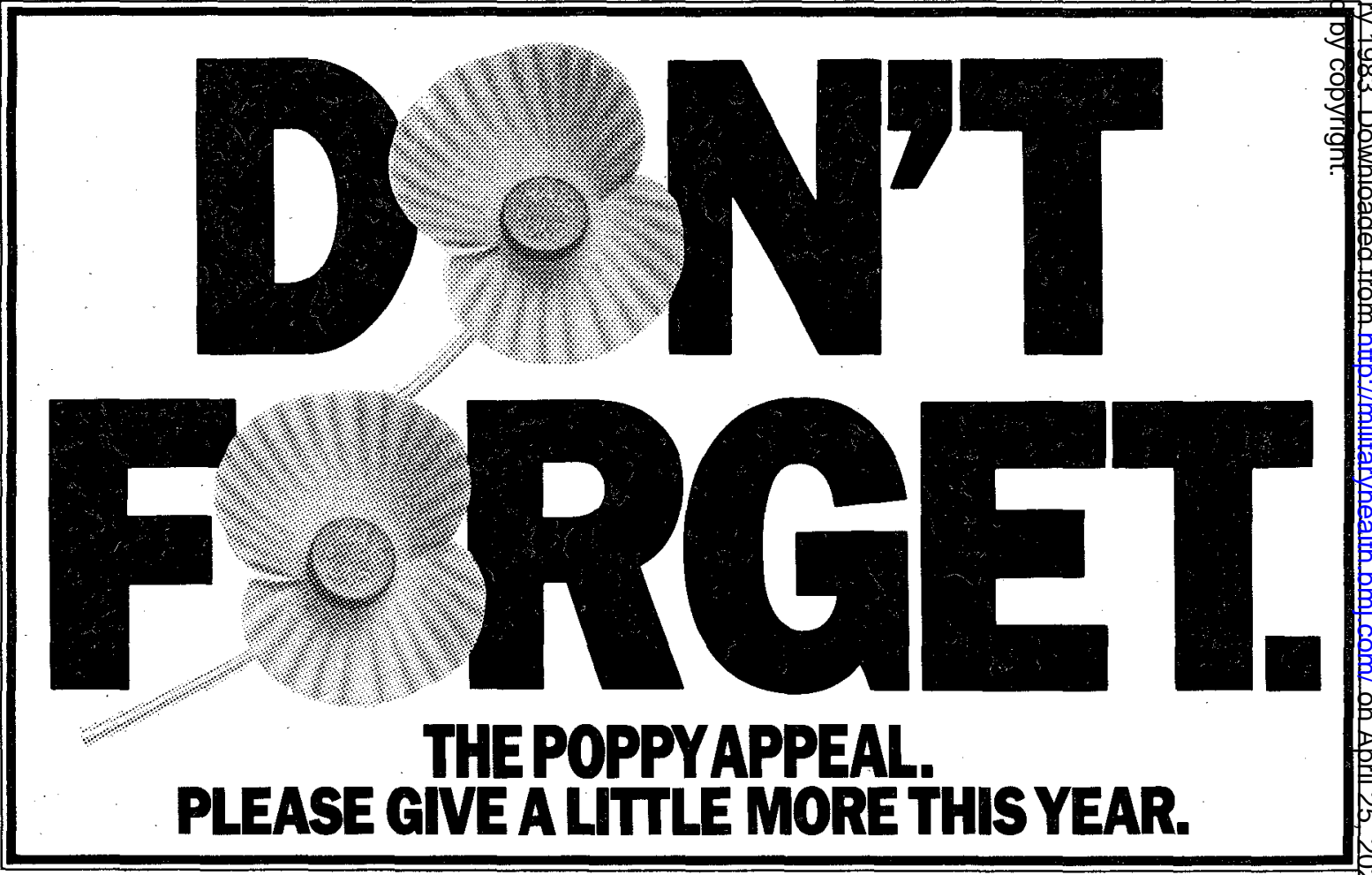

